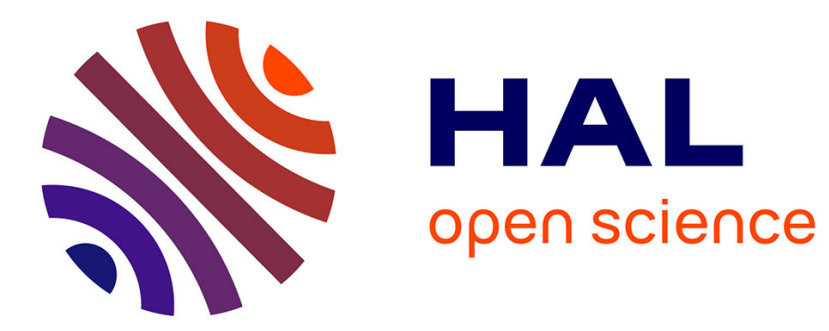

\title{
X-ray optics in Langmuir-Blodgett films
}

F. Rieutord, J.J. Benattar, Louis Bosio, P. Robin, C. Blot, R. de Kouchkovsky

\section{To cite this version:}

F. Rieutord, J.J. Benattar, Louis Bosio, P. Robin, C. Blot, et al.. X-ray optics in Langmuir-Blodgett films. Journal de Physique, 1987, 48 (4), pp.679-687. 10.1051/jphys:01987004804067900 . jpa00210485

\section{HAL Id: jpa-00210485 https://hal.science/jpa-00210485}

Submitted on 1 Jan 1987

HAL is a multi-disciplinary open access archive for the deposit and dissemination of scientific research documents, whether they are published or not. The documents may come from teaching and research institutions in France or abroad, or from public or private research centers.
L'archive ouverte pluridisciplinaire HAL, est destinée au dépôt et à la diffusion de documents scientifiques de niveau recherche, publiés ou non, émanant des établissements d'enseignement et de recherche français ou étrangers, des laboratoires publics ou privés. 
Classification

Physics Abstracts

$61.10-68.90$

\title{
X-ray optics in Langmuir-Blodgett films
}

\author{
F. Rieutord (*), J. J. Benattar $\left(^{*}\right)$, L. Bosio $\left(^{* *}\right)$, P. Robin $(* * *)$, C. Blot $\left(^{*}\right)$ \\ and R. de Kouchkovsky (*) \\ (*) DPhG/SPSRM - CEN Saclay 91191 Gif-sur-Yvette Cedex, France \\ (**) Laboratoire «Physique des liquides et électrochimie " ESPCI, 10 rue Vauquelin, 75231 Paris, France \\ (***) THOMSON CSF - Laboratoire central de recherche, Domaine de Corbeville, BP 10, \\ 91401 Orsay Cedex, France
}

(Reçu le 20 octobre 1986, accepté le 25 novembre 1986)

\begin{abstract}
Résumé. - Les milieux stratifiés et plus particulièrement les films de Langmuir-Blodgett produisent d'intéressants effets aux incidences rasantes sur les courbes de réflectivité des rayons X. Nous avons étudié la projection de la densité électronique sur la normale aux couches dans des films L.B. d'acide béhénique pour différentes séquences de dépôt, afin de corréler leur structure aux figures d'interférence particulières se produisant autour des pics de Bragg $(00 l)$. En utilisant le formalisme de l'optique et la théorie cinématique des rayons $\mathrm{X}$, nous avons analysé ces différents effets d'interférence. Par ailleurs, nous avons mis en évidence une nouvelle structure non inclinée de ces systèmes.
\end{abstract}

\begin{abstract}
Stratified media and especially Langmuir-Blodgett films give rise to interesting features of the Xray reflectivity curves at grazing incidences. We have studied the projection of the electronic density along the normal to the layers of differently sequenced L.B. Films of behenic acid, in order to correlate their structure with the particular interference patterns occurring around $(00 l)$ Bragg peaks. Using both optical formalism and kinematical theory for X-rays, we have analysed these various interference phenomena. Moreover, we found a new untilted structure of these systems.
\end{abstract}

\section{Introduction.}

$\mathrm{X}$-ray scattering techniques under grazing incidence have proved to be powerful tools for investigating the surfaces and the structure of thin films [1]. In the case of films that are made up of a few layers deposited over a thick substrate, the electronic density profile along the normal to the layers can be studied using reflectivity experiments. It has been previously shown [2] that the whole reflectivity curve displays various interesting features due to the both finite size effects and substrate influence. The aim of this paper is to analyse theoretically and experimentally the interference effects which are visible on the reflectivity pattern of stratified thin films and thus to show how information about the film deposition can be directly obtained.

Our experiments were carried out over thin organic films deposited by the classical LangmuirBlodgett (L.B.) technique [3, 4]. This technique has recently been the subject of a renewed interest since it allows the construction of various systems, having both fundamental and technological interests. (For a review see Refs. [3-5].) The data were taken on standard behenic acid layers $\left(\mathrm{C}_{22} \mathrm{H}_{44} \mathrm{O}_{2}\right)$ but the results we mention apply to any L.B. film or any regular stratified film as for example compositionmodulated amorphous thin films [6].

The interference effects we study here in detail, and which have already been observed on L.B. multilayers by a few workers, mainly consist of subsidiary maxima between main Bragg peaks. The first observation of these effects was carried out by Bisset and Iball [7]. Their interpretation of the X-ray diffraction data was only qualitative and based on the analogy with a finite size optical grating. More recently, an investigation of fine interference structure in L.B. films of manganese stearate was performed by Pomerantz et al. [2] who used both X-rays [2] and neutrons [8]. Their data processing involved a general optical formalism [9], well suited for computations but which obscured the physical origins of the observed patterns. Apart from these studies, several structure determinations using standard methods of crystallography (i.e. Fourier transforms) have been performed on L.B. films including a large 
number of layers which prevent the observation of finite size effects [10].

In this paper, we used both methods (optical formalism and Fourier transform) to analyse interference effects. Fourier transform method is less general but enables analytic calculations and easy discrimination between the origins of the pattern features. Thus we could separate in the reflectivity curve Kiessig fringes [11] and finite-size Bragg peak contributions and study the way they interfere in connection with the film deposition sequence.

The observation of such interferences was made possible only because of the very small thicknesses of the film and the high regularity of the stratification. In our case, using a high resolution device, we found that these effects were most visible on samples including about $\mathbf{3 0}$ layers corresponding to an overall thickness of $1000 \AA$ typically.

\section{Experimental.}

2.1 SAMPLE PREPARATION. - The L.B. technique for film deposition has been extensively reviewed in many places [3-5]. Let us only recall that the films are prepared by transferring floating organic monolayers onto solid substrate. In the most common deposition mode (Y type) amphiphilic molecules (i.e. molecules with hydrophilic head and aliphatic tail) stack in a tail-to-tail configuration (see Fig. 1). The orientation of the first layer strongly depends on the treatment of the substrate. The substrates we used were optically polished circular (100) silicon disks $(50 \mathrm{~mm}$ in diameter and $3 \mathrm{~mm}$ thick). When cleaned with propanol, these were found to be hydrophilic so that no deposition was observed during the first immersion. If cleaned with a dilute fluorhydric acid solution, silicon wafers become hydrophobic and aliphatic tails of monolayer bind first to the substrate. Since the deposition sequence ends by an emersion of the sample, the last layer is
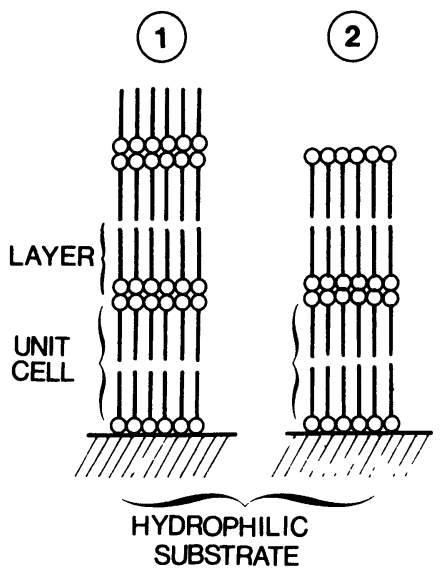
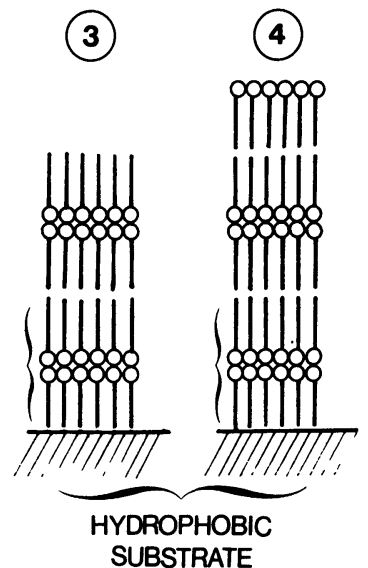

Fig. 1. - Schematic representation of the four possible deposition sequences for Y-type L.B. films. normally hydrophobic (from the aliphatic tails of the molecules) (Figs. 1-1, 1-3). Attempts were made however to end the sequence with hydrophilic heads upwards (Figs. 1-2, 1-4), by decompressing the floating monolayer before removing the substrate. Unfortunately, they result so far in poor quality samples which did not exhibit the designed features. This is certainly due to the departure of the last deposited layer during the decompression, since the hydrophilic heads were then oriented towards water.

Two series of samples corresponding to figures 1-1 and 1-3 were built with different numbers of layers. The deposition conditions were the same for all the samples ; behenic acid was spread on the surface as a solution of $10^{-3} \mathrm{~mol} / \mathrm{l}$ in chloroform. The subphase was triple distilled water (Millipore). The monolayer was kept at a constant surface pressure of $35 \mathrm{mN} \cdot \mathrm{m}^{-1}$ during deposition and the rate of transfer was $0.3 \mathrm{~cm} / \mathrm{min}$. We will later only mention the results obtained on the 26 layer and 27 layer samples, since apart from variations due to layer number, all observed features were qualitatively the same in a series.

2.2 X-RAY TECHNIQUES. - Reflectivity measurements were carried out using the experimental device which is represented in figure 2 and described in detail in reference [12]. In our geometry $\theta_{1}=\theta_{2}$ and the wavevector transfer $\left(\mathbf{Q}=\mathbf{k}_{\mathrm{f}}-\mathbf{k}_{\mathrm{i}}\right)$ is parallel to the $z$-axis perpendicular to the substrate plane (see Fig. 3). Thus we investigate here only the

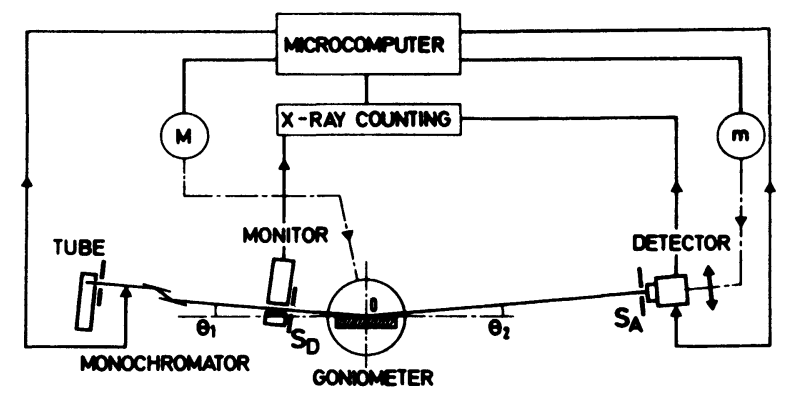

Fig. 2. - Experimental device for reflectivity measurements ; the screw mechanism driven by the motor $M$, acts on $\theta_{1}$ and $\theta_{2}$; the motor $m$ moves $\theta_{2}$ via $\mathrm{S}_{\mathrm{A}}$-detector.

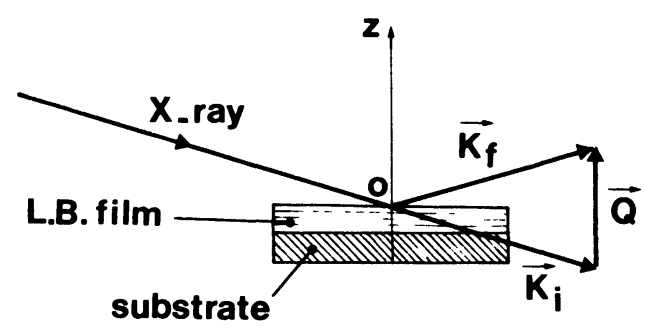

Fig. 3. - Geometry for X-ray reflectivity. The scattering vector $\mathbf{Q}$ is parallel to the $z$-axis. 
projection of the three dimensional electron density distribution onto the $z$-axis.

The X-ray source in this device is a conventional tube. The $\mathrm{Cr} \mathrm{K}_{\alpha}$ radiation is obtained from a double monochromator equipped with two flat $\operatorname{LiF}(200)$ crystals and a divergence slit $S_{D}$ which both isolates the $K_{\alpha_{1}}$ line and collimates the incident beam. The width of $S_{D}$ was $80 \mu \mathrm{m}$ and the resulting angular broadness $0.45 \mathrm{mrad}$ FWHM. The intensity of the monochromated beam is monitored by an ionization chamber while the reflected beam is detected by a scintillation counter placed behind the analysis slit $\mathrm{S}_{\mathrm{A}}$. This apparatus was employed for small angle measurements only (0-50 mrad). At higher angles, the patterns were recorded by means of classical $(\theta-2 \theta)$ goniometer using $\mathrm{V}$-filtered $\mathrm{Cr} \mathrm{K}$ radiation. In the last case, the absolute values of the reflectivity were obtained by calibrating on an overlapping range of angles scanned using the two apparatuses. The reflectance, defined as the ratio $I / I_{0}$ of reflected to incident intensities, could be measured down to $10^{-7}$.

\section{Methods for reflectivity computations.}

For X-ray wavelengths, the refractive index $n$ of matter is given by :

$$
n=1-\delta-i \beta
$$

where

$$
\begin{gathered}
\delta=\frac{\lambda^{2}}{2 \pi} r_{\mathrm{e}}\left(f+\Delta f^{\prime}\right) \frac{\rho}{M} \\
\beta=\frac{\lambda^{2}}{2 \pi} r_{\mathrm{e}} \cdot\left(\Delta f^{\prime \prime}\right)=\frac{\mu \lambda}{4 \pi}
\end{gathered}
$$

$\lambda$ is the wavelength, $r_{\mathrm{e}}=\frac{e^{2}}{m c^{2}}$ the classical electron radius, $\rho$ the density, $M$ the atomic mass, $f+$ $\Delta f^{\prime}+i \Delta f^{\prime \prime}$ the complex atomic scattering factor, $\mu$ the linear absorption coefficient. Propagation of Xrays through matter can thus be described using the laws of optics. In particular, the reflectivity of a plane diopter is given by the Fresnel formulae. Note that since the real part of the index is less than unity, total reflection will occur at an air/matter interface if the incident angle is smaller than a critical angle $\boldsymbol{\theta}_{\mathrm{c}}=\sqrt{2 \delta}$. We shall see now how to deal with a medium in which the index variès as a function of the $z$-coordinate only (so-called « stratified » medium).

3.1 MATRIX FORMALISM. - Reflectivity calculations for angles including grazing incidences require a very general method taking into account multiple reflection effects. Such a method is available from multilayer film optics $[13,14]$. The most convenient way to compute the reflectivity curve is to treat the system as a succession of homogeneous laminae. Each lamina is characterized by a transfer matrix
$M_{j}$ depending on three parameters: its thickness $d_{j}$, the refractive index $n_{j}$ and the incident angle $\theta$; $M_{j}$ is given by :

$$
M_{j}=\left(\begin{array}{c}
\cos \psi_{j}-\frac{\mathrm{i}}{p_{j}} \sin \psi_{j} \\
-i p_{j} \sin \psi_{j} \cos \psi_{j}
\end{array}\right)
$$

where $\psi_{j}=\frac{2 \pi}{\lambda} n_{j} d_{j} \sin \theta$ and $p_{j}=n_{j} \sin \theta$.

The last expression is valid for s-polarization but in the range of angles we consider, the reflectivity for the two polarizations $s$ and $p$ are about the same.

The whole medium is then represented by a characteristic matrix which is the product of the matrices of the constituting laminae. Note that for layered systems such as L.B. films, a natural intermediate stage of this calculation is the evaluation of the matrix of one layer. The reflection coefficient is given in terms of the matrix element $m_{i j}$ :

$$
r=\frac{\left(m_{11}+m_{12} p_{\mathrm{s}}\right) p_{0}-\left(m_{21}+m_{22} p_{\mathrm{s}}\right)}{\left(m_{11}+m_{12} p_{\mathrm{s}}\right) p_{0}+\left(m_{21}+m_{22} p_{\mathrm{s}}\right)}
$$

where $p_{\mathrm{s}}=n_{\mathrm{s}} \sin \theta, p_{0}=n_{0} \sin \theta$ and $n_{0}, n_{\mathrm{s}}$ are the indices of air and substrate respectively.

The final reflectivity is then :

$$
R(\theta)=\frac{I(\theta)}{I_{0}}=|r|^{2} .
$$

It should be noted that angular dependence of the atomic scattering factor (i.e. the index) can be easily included in this general formalism. The method or equivalent computation schemes [9] have been used previously in many reflectivity studies using $\mathrm{X}$-rays $[15,16]$ or neutrons $[8,17,18]$.

3.2 FOURIER TRANSFORMS. - The other method for reflectivity calculations derives from kinematical theory of diffraction. The calculation of the reflected amplitude is performed by adding all the spherical wavelets diffracted by each small volume element $\mathrm{d}^{3} r$ of the sample [19] :

$$
\mathrm{d} A=A_{0} \frac{r_{\mathrm{c}}}{r_{2}} \rho_{\mathrm{el}}(\mathbf{r}) \mathrm{d}^{3} r \exp \left(\frac{2 i \pi}{\lambda}\left(r_{1}+r_{2}\right)\right)
$$

where $A_{0}$ is the amplitude of the incident wave at the origin $0 ; r_{1}, r_{2}, r$ are the distances between the element of volume $\mathrm{d}^{3} r$ and, respectively the source, the detector and the origin $0 . \rho_{\mathrm{el}}(\mathbf{r})$ is the electronic density in the volume $\mathrm{d}^{3} r$. On account of the distances involved in reflectivity experiments, the plane wave approximation (Fraunhofer approximation) does not suffice. The problem must be treated by Fresnel diffraction. For a stratified medium, the integration over in plane coordinates $x$ and $y$ yields the expression for the reflectivity: 


$$
R=\frac{I(\theta)}{I(\theta=0)}=\left|\frac{8 \pi^{2}}{\lambda^{2} q} \int_{-\infty}^{+\infty} \delta(z) \exp (i q z) \mathrm{d} z\right|^{2}
$$

where $q=4 \pi \frac{\sin \theta}{\lambda}, \delta(z)=\frac{\lambda^{2}}{2 \pi} r_{\mathrm{e}} \rho_{\mathrm{el}}(z)$.

Note that such a calculation assumes that each element of volume sees the same incident wave and that the diffracted wave is not diffracted again (absorption and dynamical effects are neglected). Hence, the expression is valid only for $q \gg q_{\mathrm{c}}=$ $4 \pi \frac{\sin \theta_{c}}{\lambda}$.

\section{Experimental results.}

The reflectivity patterns of all the samples we studied are very similar and the main differences concern the interference phenomena occurring around the first Bragg peak. Next section will be devoted to the study of these interferences. The aim of this section is to show, through the study of a typical sample (27 layers deposited over an hydrophilic substrate), how the different parts of the reflectivity provide the information used to build a model for the electronic density profile. Three consecutive part of a reflectivity pattern are represented in figure 4.

4.1 Grazing ANGles. - The first part of this reflectivity curve (Fig. 4a) ranges between 0 and $10 \mathrm{mrad}$. For these angles, the film may be considered homogeneous with a mean refractive index $n_{\mathrm{F}}=1-\delta_{\mathrm{f}}-\beta_{\mathrm{f}}$. Measurements of the two critical angles for film and substrate $\left(\theta_{\mathrm{c}_{\mathrm{f}}}=\sqrt{2 \delta_{\mathrm{f}}}, \theta_{\mathrm{c}_{\mathrm{s}}}=\right.$ $\sqrt{2 \delta_{\mathrm{s}}}$ respectively) and the shape of the reflectivity curve at low angle, which depends mainly on absorption before $\theta_{c_{s}}$, allow determination of film and substrate mean complex indices. Fringes of equal inclination (Kiessig fringes) are already visible but their shape is much altered by the instrumental resolution. This can be observed when compared to the theoretical curve without convolution. The latter displays a narrow first minimum as well as a supplementary fringe. This shape of the pattern is due to multiple diffraction effects which become important in this angular range.

\subsection{INTERFERENCE STRUCTURE AROUND FIRST}

BRAGG PEAKS. - The second part of the reflectivity pattern (Fig. 4b) is the most interesting since it exhibits many striking features. Let us first consider the (001) Bragg peak surrounded by subsidiary maxima.

One can notice that the intensity of the subsidiary maxima is high before the first peak and very low after it. This cannot be explained by regular damping with increasing incidence, since there is about one order in magnitude between the intensities. The
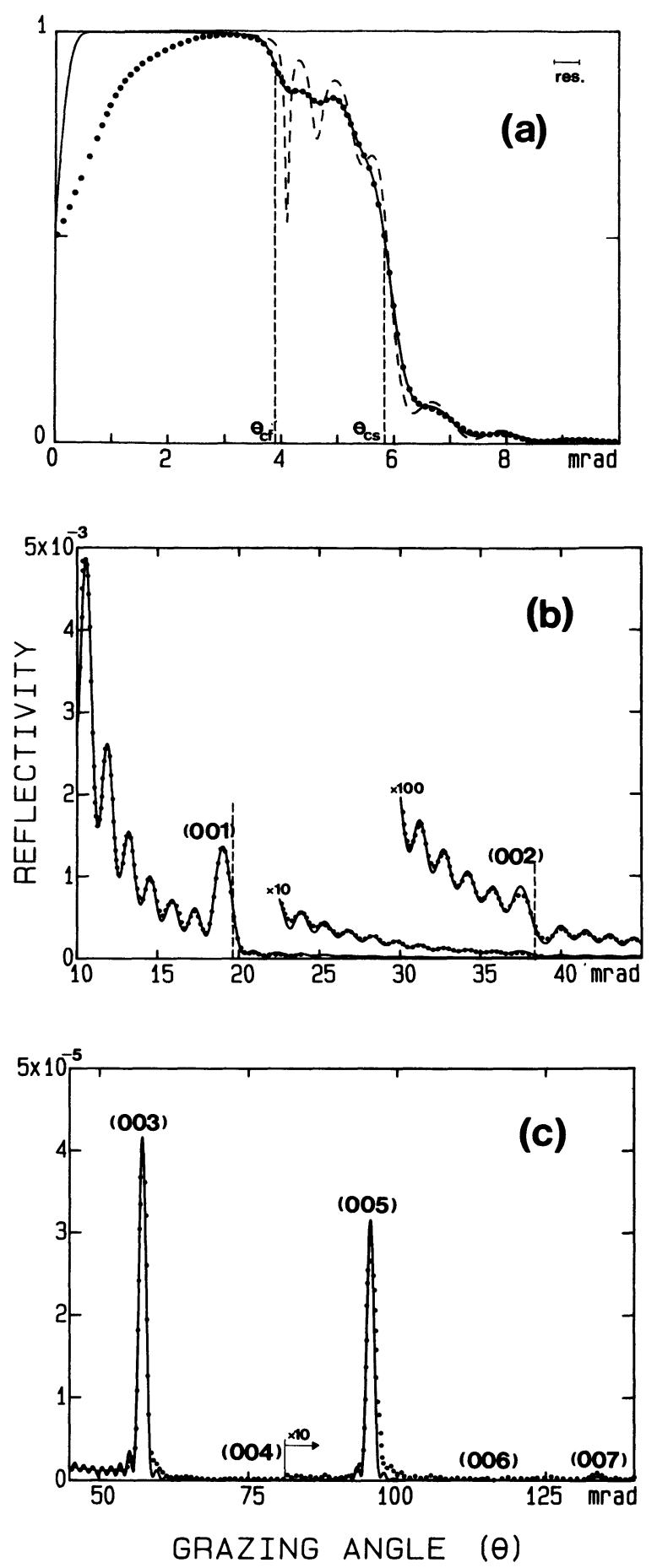

Fig. 4. - X-ray reflectivity from a 27-layer film. The dots are experimental points. The solid line corresponds to the theoretical curve computed using the model of figure 5, after convolution by experimental resolution. a) grazing angles - The dashed line is the theoretical unconvoluted curve. b) interference pattern around first and second Bragg peaks. Note the shift of the position of the (001) peak from its theoretical value (dashed line). c) higher orders Bragg peaks.

main (001) Bragg peak is clearly shifted toward small angles. The relative displacement from the position deduced from higher order peaks is $\Delta \theta / \theta \simeq 4 \%$. 
Actually, these particular effects result from interference phenomena between waves coming from two origins. Firstly, the waves reflected at the two interfaces air/film and film/substrate which would give rise, if alone, to Kiessig fringes. Secondly, waves coming from the finite-size stratified structure which yield Bragg peaks with secondary maxima. Hence, subsidiaries are a resulting pattern between Kiessig fringes and secondary Bragg peak maxima. The phase difference between the two beams depends on the relative position of interfaces and stratification (i.e. the film boundaries). Note that these interference effects which will be described further in detail in section 5, can take miscellaneous forms. For instance the dip in the reflectivity curve close to the (002) Bragg peak position is due to destructive interferences.

4.3 KIESSIG FRINGES. - Far from main Bragg peaks, subsidiaries are genuine Kiessig fringes [11]. These are fringes of equal inclination which result from the interference between the beams reflected at the air-film and film-substrate interfaces. The study of these fringes provides information relative to the film and its two interfaces. The position of the maxima of the fringes is given by the standard equation

$$
\left(1-\frac{\delta_{\mathrm{f}}}{\sin ^{2} \theta}\right) \sin \theta=p \cdot \frac{\lambda}{2 t} \quad \text { for } \quad \theta \gg \theta_{\mathrm{c}}
$$

where $t$ is the total thickness of the film, and $p$ is an integer (interference order). The term

$$
\left(1-\frac{\delta_{\mathrm{f}}}{\sin ^{2} \theta}\right)
$$

is the classical corrective term for refraction. Measurement of the position of the fringes allows an accurate determination of the total thickness of the film. In our case we found $t=815 \pm 5 \AA$.

Supplementary information can be obtained from the analysis of the fringes intensities. This kind of investigations has been performed by Croce and Nevot on thin metallic films $[20,21]$. Let us recall that rough interfaces result in additional damping of the reflectivity with increasing angles. If a Gaussian distribution is assumed for the height variations of the interface, the density profile will be represented by an error function and the reflectivity will have an additional Gaussian attenuation.

For a homogeneous film limited by two rough interfaces the intensity of the fringes on the reflectivity curve is given, from equation (1), by

$$
\begin{aligned}
& R(q)=\left(\frac{8 \pi^{2}}{\lambda^{2} q^{2}}\right)^{2} \mid \delta_{\mathrm{F}} \exp \left(-\frac{1}{2}\left\langle z_{\mathrm{AF}}^{2}\right\rangle q^{2}\right)+ \\
& +\left(\delta_{\mathrm{S}}-\delta_{\mathrm{F}}\right) \exp \left(-\frac{1}{2}\left\langle z_{\mathrm{FS}}^{2}\right\rangle q^{2}\right) \times\left.\exp (i q t)\right|^{2}
\end{aligned}
$$

where $\left\langle z_{\mathrm{AF}}^{2}\right\rangle$ and $\left\langle z_{\mathrm{FS}}^{2}\right\rangle$ are the mean squared roughnesses and $t$ is the distance between the interfaces. Thus, measurements of both mean level and amplitude of the fringes far from a Bragg peak enable a determination of these values. We find $\left\langle z_{\mathrm{AF}}^{2}\right\rangle^{1 / 2}=20 \AA$ and $\left\langle z_{\mathrm{FS}}^{2}\right\rangle^{1 / 2}=5 \AA$. The roughness of the air-film interface is rather important and indicates that the last layer should be poorly filled. This fact is in agreement with a previously expected mechanism $[22,23]$ and with our observations made by electron microscopy on replicas [24]. Micrographs taken on these samples look rather blurred, on account of the disorder of the last layer. These features are probably dependent also on the film structure which we shall examine now.

4.4 Structure AlONG THE NORMAL TO THE LAYERS. - Bilayer spacing can be easily obtained from the last part of the pattern (Fig. 4c). Here, only main Bragg peaks are visible, subsidiaries being drowned in the background scattering. Interlamellar periodicity obtained from Bragg peaks up to (007) was found to be : $d_{001}=60.0 \pm 0.1 \AA$. This result is surprising since it corresponds precisely to twice the length of a behenic acid molecule, thus involving untilted disposition of molecules within the unit cell. To our knowledge, structures observed so far in L.B. films of behenic acid were similar to known bulk crystalline structures of long normal chain carboxylic acid ; all these forms (A, B or C) involve non zero tilt angles $[25,26]$. Note that, since the film thickness $(815 \AA)$ corresponds to 27 times the layer thickness $(60.0 \AA / 2)$, then all the layers have the same structure.

The electronic density profile within a unit cell can be obtained from the analysis of the Bragg peak intensities (i.e. the structure factor). The first feature which can be noticed is the very weak amplitude of even order Bragg peaks. This results from both excess and lack of electrons due to hydrophilic heads (-COOH groups) and interchain spacing respectively, separated by half a period [2, 27]. It should be pointed out that the structure factor decreases strongly and only seven orders can be observed. For a comparison we observed up to 24 orders in films of $\mathrm{C}$-form structure under similar conditions. This means that the electronic density profile within the unit cell is rather smooth. This fact may indicate that this system should be a stacking of two dimensional layers weakly correlated.

Another argument for that was suggested by observation of the surface by means of electron microscopy on replicas. Contrary to multilayers of $\mathrm{C}$-form behenic acid, their surfaces do not exhibit multistep defects (extra islands of holes) [24] but look rather blurred.

Using the formalism given in section 3.1, the reflectivity curve can be calculated if the whole index 
profile $\delta(z)$ is known. The reflectivity curve calculated using the $\delta(z)$ profile given in figure 5 (solid line in Fig. 4) is found to fit the experimental data very well, after convolution by the instrumental resolution. The fitted values found for $\delta(z)$ are close to the theoretical ones, calculated by taking into account the steric hindrance, atomic composition and standard distances between atoms. The absorption for this kind of layers is very small $\left(\beta \sim 2 \times 10^{-8}\right)$ and can be neglected for angles greater than the critical angle. Concerning the layer structure, one can assume, since the molecules are not tilted, that their lateral stacking within a layer is hexagonal.

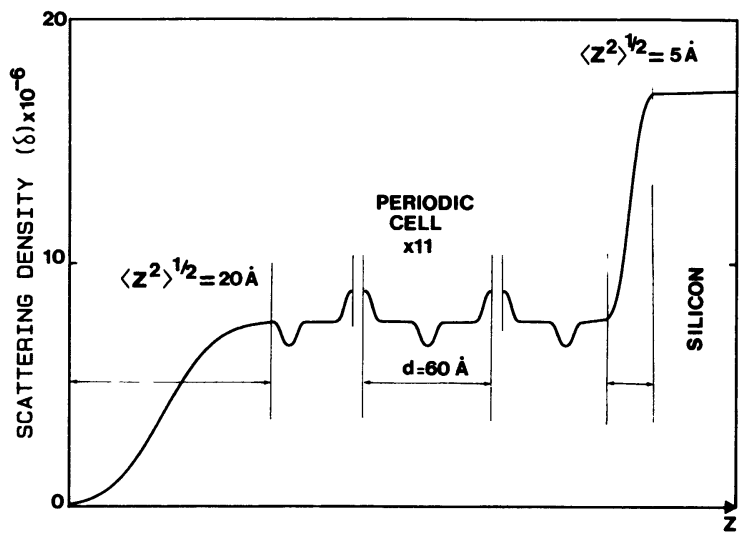

Fig. 5. - Model of the index profile for the 27-layer sample along the $z$-axis. The mean value of the real part of the index of the film is $\delta_{\mathrm{f}}=7.6 \times 10^{-6}$ and the corresponding imaginary part $\beta_{\mathrm{f}}=2 \times 10^{-8}$. For the silicon substrate : $\delta_{\mathrm{s}}=17.1 \times 10^{-6}$ and $\beta_{\mathrm{s}}=0.8 \times 10^{-6}$.

\section{Calculation of the interference structure.}

The previous section showed that particular interference features are visible at small angles on the reflectivity curve. The main drawback of the optical matrix formalism (Sect. 3.1) is that, concerning our systems, it does not show the origins of the different features on the reflectivity pattern. Fortunately, for angles greater than a few $\boldsymbol{\theta}_{\mathrm{c}}$, multiple diffraction effects are weak (reflectivities are small) so that kinematical approximation (i.e. single scattering) is valid (Sect. 3.2).

Analytic expressions can therefore be obtained, showing clearly the effects we aim to discuss. We shall assume that the systems are composed of identical monolayers. For the sake of clarity, calculations will be made in all cases with the same structure within the unit cell and with step-like interfaces. The four cases of figure 1 will be reviewed.

5.1 EVEN NUMBER OF LAYERS. - The easiest case deals with films including even numbers of deposited layers since these are periodic systems with an integer number $(N)$ of bilayers. The index profile is represented by a periodic function of period $d$ (bilayer spacing) between $z=0$ and $z=-N d$. Setting $\delta(z)=\left(\delta(z)-\delta_{\mathrm{f}}\right)+\delta_{\mathrm{f}}$ where $\delta_{\mathrm{f}}$ is the mean value of the index within the film, one gets, performing the Fourier transform (1) :

$$
\begin{gathered}
R(q)=\left(\frac{8 \pi^{2}}{\lambda^{2} q}\right)^{2} \times\left[\frac{\left(\delta_{\mathrm{s}}-2 \delta_{\mathrm{f}}\right)^{2}}{q^{2}}+\frac{4 \delta_{\mathrm{f}}\left(\delta_{\mathrm{s}}-\delta_{\mathrm{f}}\right)}{q^{2}} \times\right. \\
\times \cos ^{2}\left(\frac{N q d}{2}\right)+F^{2} \frac{\sin ^{2}\left(N q \frac{d}{2}\right)}{\sin ^{2}\left(q \frac{d}{2}\right)} \\
\left.+2 F \frac{\left(2 \delta_{\mathrm{f}}-\delta_{\mathrm{s}}\right)}{q} \frac{\sin ^{2}\left(N q \frac{d}{2}\right)}{\sin \left(q \frac{d}{2}\right)}\right]
\end{gathered}
$$

where $F(q)$ is defined as :

$$
F=\int_{-d / 2}^{d / 2}\left(\delta(z)-\delta_{\mathrm{f}}\right) \exp (i q z) \mathrm{d} z
$$

( $F$ is proportional to the structure factor for the unit cell.)

It is assumed from the deposition mode that the cell is centrosymmetric so that $F$ is real. $F$ is positive in case 3 of figure 1 and negative in case 2 .

The leftmost two terms in the brackets in equation (3) are the Kiessig fringes contribution. The expression is the same as equation (2) where the roughnesses have been taken to be equal to zero.

The third term corresponds to Bragg peaks with secondary maxima. Their intensity is proportional to the squared structure factor. The expression is exactly the same as for a finite size optical grating.

The rightmost term is the interference term between the two previous phenomena. In the present case, this term is small since $\left(2 \delta_{\mathrm{f}}-\delta_{\mathrm{s}}\right)$ is small : the mean density of the film $\left(\delta_{\mathrm{f}}=7.6\right)$ is close to half the density of silicon $\left(\delta_{\mathrm{s}}=17.1\right)$.

Figure 6 shows a plot of separate contributions of Kiessig fringes and of (001) Bragg peak, then the result of all the terms of equation (3). Due to the small interference term, intensities merely add and there is only a slight difference between the cases $(F<0)$ and $(F>0)$. Note that Kiessig fringes have the same spacing as secondary Bragg peaks, but are shifted by half a period. Fringes on both sides of the peaks have about the same amplitude, and no significant shift of the main Bragg peak can be detected.

5.2 ODD NUMBER OF LAYERS. - The calculations are in this case somewhat more tedious since the structure is no longer periodic (cases 1 and 4 in 
Fig. 1). The simplest way to tackle this problem is to consider the system as a succession of $(N)$ bilayers and an additional monolayer (the last layer in the deposition sequence for instance). This last layer can be replaced by a homogeneous lamina having the mean index of the film : this includes its contribution to the phase difference between the reflected waves, but neglects its small contribution to Bragg peak intensity. Under this assumption, the Fourier transform becomes :

$$
\begin{aligned}
& R(q)=\left(\frac{8 \pi^{2}}{\lambda^{2} q}\right)^{2} \times\left[\frac{\left(\delta_{\mathrm{s}}-2 \delta_{\mathrm{f}}\right)^{2}}{q^{2}}+\frac{4 \delta_{\mathrm{f}}\left(\delta_{\mathrm{s}}-\delta_{\mathrm{f}}\right)}{q^{2}} \cos ^{2}\left(\left(N+\frac{1}{2}\right) \frac{q d}{2}\right)+F^{2} \frac{\sin ^{2}\left(N \frac{q d}{2}\right)}{\sin ^{2}\left(\frac{q d}{2}\right)}+\right. \\
& \left.+2 F \frac{\sin \left(N q \frac{d}{2}\right)}{\sin \left(q \frac{d}{2}\right)} \times \frac{1}{q}\left(\left(\delta_{\mathrm{f}}-\delta_{\mathrm{s}}\right) \sin \left(\frac{(N+1) q d}{2}\right)+\delta_{\mathrm{f}} \sin \left(\frac{N q d}{2}\right)\right)\right]
\end{aligned}
$$

with the same notation as in (3).

The value of $\frac{q d}{2}$ corresponding to a $(00 l) \mathrm{Bragg}$ peak is $l \pi$. It can be seen that the last interference term is the sum of two opposite terms for even $l$ and the difference of these terms for odd $l$. As the absolute values of these terms are close to each other, the interference term is small in one case (even $l$ ) but large in the other (odd $l$ ).

Figure 7 shows the interference effect around the (001) Bragg peak. In this case, fringes and secondary maxima coincide, so that the interference has a very large effect on the intensity of subsidiaries. The sign change of the large interference term from one side of the Bragg peak to the other results in a very strong damping of the fringes. The ratio of their intensity before and after the peak is actually about 100. The calculations predict that this striking effect should be inverted in the case of a deposition over an hydrophobic substrate (Fig. 1-4) since this case fol-

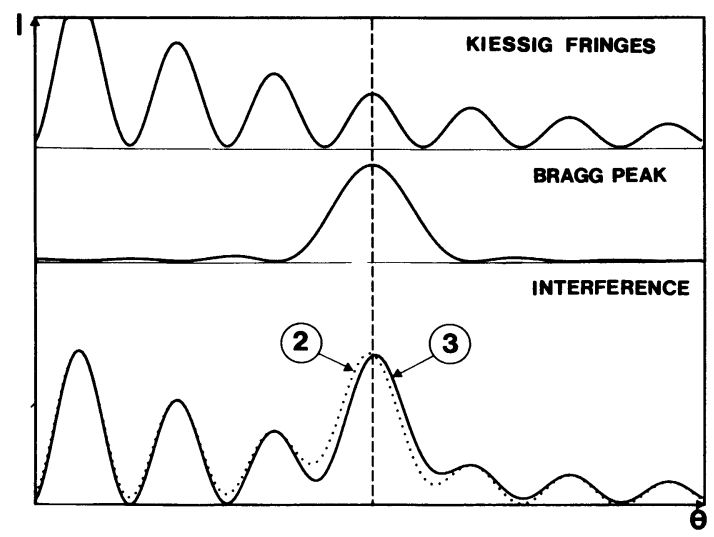

Fig. 6. - Interference between Kiessig fringes and subsidiary maxima of the (001) Bragg peak for an even number of layers. The data used in the calculation correspond to behenic acid, assuming no roughnesses nor absorption. Numbers 2 and 3 refer to cases 2 and 3 in figure 1 .

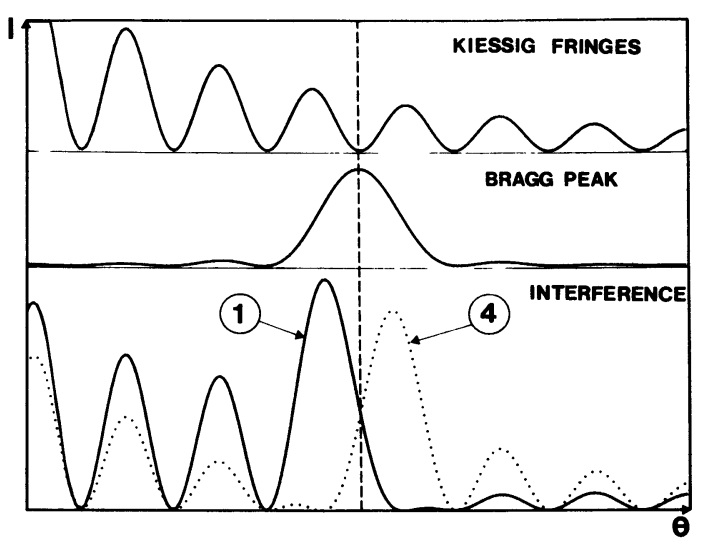

Fig. 7. - Interference structure around (001) for odd number of layers. Numbers 1 and 4 refer to cases 1 and 4 in figure 1 .

lows from the previous one by solely changing the term $F$ into $-F$.

The unusual shape for the main Bragg peak is also directly explained by this interference process : a fringe is added on the low angle side of the main peak and subtracted on the other side. The amplitude of the displacement of the peak maxima depends on the relative heights of Kiessig fringes and Bragg peaks (i.e. on $N, F, \delta_{F}, \delta_{s}$ ). In our example in figure 7 , the shift of the maxima is $\frac{\Delta \theta}{\theta}=4 \%$ and the intensity change by a factor of two. Note that we also computed the interference pattern of figures 6-7 using matrix formalism. Apart from a small angular shift due to the refraction, no difference with the plot of equations (3) and (4) was detected.

5.3 EXPERIMENTAL INTERFERENCE PATTERN. Figure 8 shows the experimental reflectivity data around the (001) Bragg peak for different sequences of deposition. Figure 8a displays the reflectivity for a 26-layer sample with hydrophobic substrate (case 3 


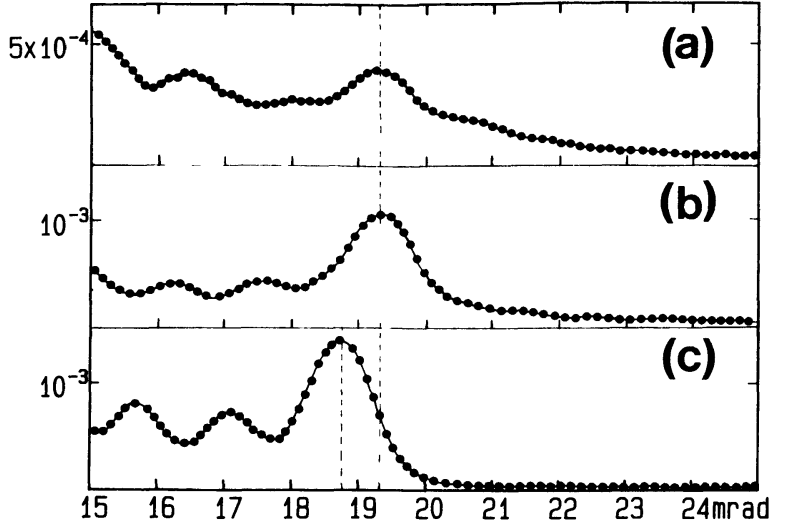

Fig. 8. - Experimental results for the interference structure around (001) Bragg peak. a) 26-layer sample on hydrophobic substrate (case 3 in Fig. 1), b) Sample planned to be 27-layer on hydrophobic substrate (case 4 in Fig. 1). The last layer actually went off so that the sequence is the same as for the 26-layer sample. c) 27 layer sample on hydrophilic substrate - case 1 in figure 1.

in Fig. 1). The main features that we mentioned in section 5.1 are observed : there is no special damping of the subsidiaries from one side of the (001) Bragg peak to the other one and no important shift of the position from the theoretical value. It should be noted however that the intensities of both Kiessig fringes and Bragg peaks are much smaller for the 26layer than for the 27 -layer sample. This originates in the substrate treatment, which increases its roughness and changes the quality of layer deposition. Apart from this overall damping, the structure factor remains the same.

Figure $8 \mathrm{~b}$ displays the same angular range for a sample which was designed to include 27 layers deposited over an hydrophobic substrate (i.e. case 4 in Fig. 1). The curve does not show the expected feature but has a similar shape as the previous one. This indicates that the last layer went off during the final decompression. This fact is also supported by a measurement of the film thickness which indicates less than 27 layers. For the same reasons, similar problems were encountered on samples corresponding to the case 2 in figure 1 . (Even number of layers on hydrophilic substrates.) The patterns we obtained were typical of odd number samples on hydrophilic substrates (case 1 of Fig. 1). Figure 8c reproduces an example of such patterns taken from figure $4 \mathrm{~b}$ (27 layer sample); all the features described in section 5.2 and plotted in figure 7 are visible.

\section{Conclusion.}

Interference phenomena play a major role in X-ray reflectivity studies of layered thin films. We saw that these effects are large in the small angle part of the reflectivity curve and that they strongly alter classical diffraction features. These effects, which must be taken into account in structure factor determination, may be used to investigate the boundaries of the film : the deposition sequence, i.e. the orientation of the first and the last layer may be straightforwardly determined. The method is actually more general : we illustrate here only cases differing from each other by the presence of one additional layer but it is clear that other cases involving a layer with tilted molecules, a half filled layer and so on are possible. All will change the phase difference between the waves coming from the boundaries and those coming from the structure itself; thus the interference pattern will be modified and one can take advantage of this fact to set one with respect to the other.

This kind of investigation can be performed ideally on L.B. films since the L.B. technique allows the deposition of a given number of highly regular layers. Our results obtained on behenic acid enabled us to describe a new untilted form for this molecule as well as to confirm various observations on L.B. films (i.e. mainly the existence of a disordered last layer). Further experiments on new samples built under different conditions (especially to prevent the departure of the last layer when hydrophilic) will be carried out. Moreover this method may be applied to the study of overturning mechanism and X-type layers.

X-ray reflectivity study of many other systems, such as composition modulated amorphous thin films or MBE grown layers [28], may also be very interesting, since a very large variety of artificial structures may be designed with these techniques.

\section{Acknowledgments.}

We wish to thank E. Chastaing for technical assistance and M. Dupeyrat for helpful discussions.

\section{References}

[1] Rieutord, F., Benattar, J. J. and Bosio, L., $J$. Physique 47 (1986) 1249.

[2] Pomerantz, M. and Segmüller, A., Thin Solid Films 68 (1980) 33.

[3] Roberts, G. G., Adv. Phys. 34 (1985) 475.
[4] - Proceedings of the 1st Int. Conf. on L. B. films Thin Solid Films (1982).

- Thin Solid Films 99 (1983) and 68 (1980).

[5] GAINES, G. L., Insoluble Monolayers at Liquid-Gas Interfaces (Interscience, New York) 1966. 
[6] Bruson, A., Piecuch M. and Marchal, G., J. Appl. Phys. 58 (1985) 1229.

[7] Bisset, D. C. and Iball, J., Proc. Soc. London Sect. A 67 (1954) 315.

[8] Nicklow, R. M., Pomerantz, M., Segmüller, A., Phys. Rev. B 23 (1981) 1081.

[9] Parrat, L. G., Phys. Rev. 95 (1954) 359.

[10] Lesslauer, W., Acta Cryst. B 30 (1974) 1927.

[11] Kiessig, H., Ann. Phys. 10 (1931) 769.

[12] Bosio, L., Cortès, R., Folcher, G., Oumezine, M., Revue Phys. Appl. 20 (1985) 437.

[13] Abeles, F., Ann. de Physique 5 (1950) 596.

[14] Born, M. and Wolf, E., Principles of Optics (Pergamon Press) (1984) 6th edition p. 51.

[15] Lu, B. C. and Rice, S. A., J. Chem. Phys. 66 (1978) 5558.

[16] Bosio, L. and Oumezine, M., J. Chem. Phys. 80 (1984) 954.

[17] Hayter, J. B., Highfield, R. R., Pullman, B. J., Thomas, R. K., McMullen, A. I. and PenFOLD, J., J. Chem. Soc. Faraday Trans. 77 (1981) 1437.
[18] Highfield, R. R., Thomas, R. K., Cummins, P. G., GREGORY, D. P., MingINS, J., HAYTER, J. B., SCHÄRPF, O., Thin Solid Films 99 (1983) 165.

[19] See e.g. Warren B. E., X-ray diffraction. (AddisonWesley) 1969.

[20] Croce, P. and Nevot, L., Revue Phys. Appl. 11 (1976) 113.

[21] Nevot, L. and Croce, P., Revue Phys. Appl. 15 (1980) 761.

[22] Hasmonay, H., Vincent, M. and Dupeyrat, M., Thin Solid Films 68 (1980) 21.

[23] Honig, E. P., J. Coll. Interf. Sci. 43 (1973) 66.

[24] Allain, M., Benattar, J. J., Rieutord, F. and RobIN, P., Europhys. Lett. to be published.

[25] Abrahamson S. and von Sydow, E., Acta Cryst. 7 (1954) 591.

[26] von Sydow, E., Acta Cryst. 8 (1955) 557.

[27] Prins, J. A., Physica 6 (1926) 35.

[28] Chang, L. L., Segmüller, A. and Esaki, L., Appl. Phys. Lett. 28 (1975) 39. 